\title{
ALMOST RECURSIVELY ENUMERABLE SETS
}

\author{
BY
}

JOHN W. BERRY( $\left.{ }^{1}\right)$

\begin{abstract}
An injective function on $N$, the nonnegative integers, taking values in $N$, is called almost recursive (abbreviated a.r.) if its inverse has a partial recursive extension. The range of an a.r. function $f$ is called an almost recursively enumerable set in general; an almost recursive set if in addition $f$ is strictly increasing. These are natural generalizations of regressive and retraceable sets respectively. We show that an infinite set is almost recursively enumerable iff it is point decomposable in the sense of McLaughlin. This leads us to new characterizations of certain classes of immune sets. Finally, in contrast to the regressive case, we show that a.r. functions and sets are rather badly behaved with respect to recursive equivalence.
\end{abstract}

1. Introduction and notation. Almost recursive functions, almost recursive sets and almost recursively enumerable sets were introduced by Vučković in [7]. For basic facts about retraceable sets the reader is referred to [3]; for regressive sets to [2] and [1].

Throughout this paper, $N$ denotes the set of nonnegative integers. The word "set" will usually mean "subset of $N$," and the complement $N-A$ of a set $A$ is denoted by $\bar{A}$. If $f$ is a partial function on $N$ we denote its domain and range by $\delta(f)$ and $\rho(f)$ respectively. $\pi_{e}$ denotes the eth partial recursive function in the Kleene enumeration, i.e. $\pi_{e}(x) \cong U\left(\mu y T_{1}(e, x, y)\right)$. $\omega_{e}$ denotes $\delta\left(\pi_{e}\right)$. As a pairing function we use $j(x, y)=\frac{1}{2}\left[(x+y)^{2}+3 y+x\right]$, and $k(x), l(x)$ are recursive functions which satisfy $x=j(k(x), l(x))$ for all $x$, and $k(j(x, y))=x, l(j(x, y))=y$ for all $x$ and $y$. $\operatorname{Pr}(n)$ denotes the $n$th prime number $(\operatorname{Pr}(0)=1, \operatorname{Pr}(1)=2$, etc.). We shall use an effective listing $D(x)$ of finite subsets of $N$ defined as follows: if $x_{1}, x_{2}, \ldots, x_{n}$ are distinct elements of $N$ and $x=2^{x_{1}}+2^{x_{2}}+\cdots+2^{x_{n}}$ then $D(x)=\left\{x_{1}, x_{2}, \ldots, x_{n}\right\}$, and $D(0)=\varnothing$. Let $f$ be a recursive function. The sequence of sets $\omega_{f(0)}, \omega_{f(1)}, \ldots$ is called a disjoint array if $\omega_{f(x)} \neq \varnothing$ for every $x$, and $x \neq y \Rightarrow \omega_{f(x)} \cap \omega_{f(y)}=\varnothing$ for all $x$ and $y$. If, in addition, $\omega_{f(x)}$ is a finite set for every $x$, then the sequence is called a finite disjoint array. A sequence of finite sets $D(f(0)), D(f(1)), \ldots$ (again for some

Received by the editors April 20, 1970 and, in revised form, February 17, 1971.

AMS 1969 subject classifications. Primary 0270; Secondary 0272, 0274, 0275.

Key words and phrases. Almost recursive set, almost recursively enumerable set, retraceable set, regressive set, hyperhyperimmune set, recursively enumerable sequences, discrete arrays, recursive equivalence types.

(1) These results are part of the author's doctoral dissertation presented to the University of Notre Dame in partial fulfillment of the requirements for the degree of Doctor of Philosophy. The author would like to thank Dr. V. Vučković for his advice and encouragement during its preparation, and the referee for suggesting the construction of Theorem 6.4.

Copyright (C) 1972, American Mathematical Society 
recursive $f$ ) is called a discrete array if $D(f(x)) \neq \varnothing$ for every $x$, and $x \neq y \Rightarrow D(f(x))$ $\cap D(f(y))=\varnothing$ for every $x$ and $y$. A disjoint array meets a set $A$ if $\omega_{f(x)} \cap A \neq \varnothing$ for every $x$; similarly for discrete arrays. For convenient reference we set up the following notations for various classes of sets:

$R E T=$ the class of all retraceable sets

$R E G=$ the class of all regressive sets

$A R=$ the class of all almost recursive sets

$A R E=$ the class of all almost recursively enumerable sets

$I=$ the class of all immune sets

$H I=$ the class of all hyperimmune sets

$H H I=$ the class of all hyperhyperimmune sets

$S H I=$ the class of all strongly hyperimmune sets

$S H H I=$ the class of all strongly hyperhyperimmune sets

$F S H I=$ the class of all finitely strongly hyperimmune sets

\section{Basic definitions and results.}

Definition 2.1. An injective total function $a$ on $N$ to $N$ is called almost recursive (abbreviated a.r.) if there is a partial recursive function $F$ such that $\delta(F) \supset \rho(a)$ and $F(a(n))=n$ for every $n \in N$ (i.e., if $a^{-1}$ has a partial recursive extension). A set $A$ is called almost recursive $(A \in A R)$ if $A$ is finite or the range of a strictly increasing a.r. function. $A$ is called almost recursively enumerable $(A \in A R E)$ if $A$ is finite or the range of an a.r. function. Note that our definition of the class $A R E$ differs slightly from that given by Vučković at the end of [7], but it is easily shown that the two are equivalent.

It is clear that $A R \subset A R E$, that $R E T \subset A R$ and that $R E G \subset A R E$. Note also that Vučković has proved [7, Theorem 2.1] that if $A \in A R$ and $\bar{A}$ is recursively enumerable, then $A \in R E T$. $A R E$ is a rather large class of sets. If $f$ is any partial function let $G_{f}=\{j(x, y) \mid x \in \delta(f) \wedge f(x)=y\}$. Then it is easily shown that $G_{f} \in A R E$ for any partial function whose domain is recursively enumerable, in particular, for any total function whatsoever. Furthermore, $A R E$ contains sets in each of the Uspenskii classes $\mathscr{B}_{0}$ to $\mathscr{B}_{4}$ [6, p. 120]. In [7], Vučković has given examples of $A R$ sets in classes $\mathscr{B}_{0}, \mathscr{B}_{1}, \mathscr{B}_{2}$ and $\mathscr{B}_{4}$. So it only remains to construct an $A R E$ set in $\mathscr{B}_{3}$.

\section{THEOREM 2.1. $A R E \cap \mathscr{B}_{3} \neq \varnothing$.}

Proof. Let $A$ be an immune retraceable set such that $\bar{A}$ is recursively enumerable, and put $B=j(N \times A)$. If $p_{A}$ denotes the principal function of $A$ (i.e., that strictly increasing function whose range is $A$ ) then $g(x)=j\left(k(x), p_{A}(l(x))\right)$ is an injective function with range $B$. Let $\varphi$ be a partial recursive extension of $p_{A}^{-1}$. Then $F(y) \simeq j(k(y), \varphi(l(y)))$ is a partial recursive extension of $g^{-1}$, so $B \in A R E$. It is well known that $j(N \times A) \in \mathscr{B}_{3}$ for any cosimple set $A$ (see e.g. [6, p. 120]). Hence $B \in A R E \cap \mathscr{B}_{3}$. 
In [1], Appel \& McLaughlin have proved that if $A$ and $\bar{A}$ are regressive, then at least one of them must be recursively enumerable. Such a property fails to extend to $A R E$ (even to $A R$ ) in the strongest possible manner.

Definition 2.2. For any set $A$, denote by $S(A)$ the set

$$
\{2 x \mid x \in A\} \cup\{2 x+1 \mid x \notin A\} .
$$

That is, $S(A)=A$ join $\bar{A}$.

THEOREM 2.2. Let $A$ be any subset of $N$. Then

(i) $A \equiv{ }_{T} S(A)$,

(ii) $S(A) \in A R$,

(iii) $(S(A))^{-}=S(\bar{A})$.

Proof. For (i), it is easily shown that $A \leqq_{1} S(A)$ and that $S(A) \leqq_{T} A$. For (ii), note that $F(y)=\left[\frac{1}{2} y\right]$ (where $[x]$ is the greatest integer function) is a partial recursive extension of $p_{S(A)}^{-1}$. (iii) is a trivial computation.

COROllary 2.3. Every Turing degree contains a set $B$ such that both $B$ and $\bar{B}$ belong to $A R$.

3. Two characterizations of almost recursively enumerable sets.

Definition 3.1. A partial recursive function $\varphi$ of two variables is called admissible if

(i) $\bigwedge_{i} \bigvee_{y}[\varphi(i, y)$ is defined $]$, and

(ii) $\bigwedge_{i} \bigwedge_{j} \bigwedge_{x} \bigwedge_{y}[i \neq j \Rightarrow \varphi(i, y) \neq \varphi(j, z)]$.

If $\varphi$ is an admissible function, define sets $\Phi_{i}$ for each $i$ by

$$
\Phi_{i}=\{y \mid \varphi(i, y) \text { is defined }\} .
$$

THEOREM 3.1. Let $a(x)$ be a total injective function on $N$. Then $a(x)$ is an a.r. function if and only if there exists an admissible function $\varphi_{a}$ and a total injective function $h$ such that

(i) $\bigwedge_{i}\left[h(i) \in \Phi_{i}\right]$, and

(ii) $\bigwedge_{i}\left[a(i)=\varphi_{a}(i, h(i))\right]$.

Proof. Suppose first that $a$ is an almost recursive function and let $F$ be a partial recursive extension of $a^{-1}$. For each $i$ define $\Phi_{i}=\{y \mid y \in \delta(F) \wedge F(y)=i\}$. Put $\varphi_{a}(i, y)=y$ for all $y \in \Phi_{i}$. Then $\varphi_{a}$ is a partial recursive function which is clearly admissible. Putting $h(i)=a(i)$ for all $i$, the conditions of the theorem are satisfied.

Conversely, suppose we are given functions $\varphi_{a}$ and $h$ as in the hypothesis. Define the sets $\Phi_{i}$ for $\varphi_{a}$ as in Definition 3.1, and use these to define a partial recursive function $F$ by setting $F\left(\varphi_{a}(i, y)\right)=i$ for all $y \in \Phi_{i}$. Then $F$ is a partial recursive extension of $a^{-1}$ and hence $a$ is an a.r. function.

THEOREM 3.2. Let $A$ be an infinite set. Then $A \in A R E$ if and only if there exists a recursive function $f$ such that $\left\{\omega_{f(x)}\right\}$ is a disjoint array satisfying:

(i) $\bigwedge_{x}\left[\omega_{f(x)} \cap A\right.$ is a singleton $]$ and

(ii) $A \subset \bigcup_{x \in N} \omega_{f(x)}$.

(We say that such an array witnesses the fact that $A \in A R E$.) 
Proof. First suppose that $A \in A R E$ and let $a$ be an a.r. function with $\rho(a)=A$. Let $F$ be a partial recursive extension of $a^{-1}$. Define a sequence of sets $E_{i}$ by setting

$$
x \in E_{i} \Leftrightarrow F(x)=i .
$$

This is a recursively enumerable predicate so there exists a recursive function $f$ such that $E_{i}=\omega_{f(i)}$ for every $i$. Clearly $\left\{\omega_{f(i)}\right\}$ is a disjoint array satisfying (i) and (ii).

For the converse suppose that we are given a disjoint array $\left\{\omega_{f(x)}\right\}$ satisfying (i) and (ii). Define a function $a$ by setting $a(n)=$ the unique element of $\omega_{f(n)} \cap A . a(n)$ is well defined for every $n$ by (i), and is injective by the disjointness of the array. Now define a partial recursive function $F$ by

$$
F(x)=i \Leftrightarrow x \in \omega_{f(i)} .
$$

Disjointness of the array guarantees that $F$ is well defined. $F$ is clearly a partial recursive extension of $a^{-1}$, and hence $\rho(a)=A$ is in $A R E$.

In [5], McLaughlin defined a set $A$ to be point decomposable if there is a disjoint array satisfying (i) and (ii) above. Thus the point decomposable sets are exactly the infinite almost recursively enumerable sets.

We shall repeatedly need the following result, which is implicit in Theorem 6 of [8].

TheOREM 3.3 (YATES). Let $A$ be an infinite set. Then the following two statements are equivalent:

(i) A has no infinite retraceable subset.

(ii) There is no recursive function $f$ such that $\left\{\omega_{f(x)}\right\}$ is a disjoint array which meets $A$.

The class of infinite sets with these two properties is $S H H I$. Note that the implication (ii) $\Rightarrow$ (i) follows at once from our Theorem 3.2.

THEOREM 3.4. Every infinite set in ARE contains an infinite retraceable subset.

Proof. We note that this result follows at once from Theorems 3.2 and 3.3 It is instructive, however, to observe that it can also be proved directly by a simple technique which bypasses Yates' theorem. Namely, let $a$ be an a.r. function with range $A$, and $F$ a partial recursive extension of $a^{-1}$. Define $a^{n}(x)$ by $a^{0}(x)=x$; $a^{n+1}(x)=a\left(a^{n}(x)\right)$. If $A=N$ the theorem is trivially true, so suppose $A \neq N$ and consider the set: $A_{x}=\left\{a(x), a^{2}(x), \ldots\right\}$ for any $x \notin A$. This is clearly an infinite regressive subset of $A$ and hence, by Proposition 7 of [2], contains an infinite retraceable subset.

THEOREM 3.5. Let $A$ and $B$ be separable sets in $A R E$. Then $A \cup B \in A R E$.

Proof. Let $E_{1}$ and $E_{2}$ be recursively enumerable sets such that $E_{1} \cap E_{2}=\varnothing$, $A \subset E_{1}$ and $B \subset E_{2}$. Let $\left\{\omega_{f(x)}\right\}$ and $\left\{\omega_{g(x)}\right\}$ be disjoint arrays witnessing $A \in A R E$ and $B \in A R E$, respectively. Let $f^{\prime}$ and $g^{\prime}$ be recursive functions such that, for every $x$, 
$\omega_{f^{\prime}(x)}=\omega_{f(x)} \cap E_{1}$ and $\omega_{g^{\prime}(x)}=\omega_{g(x)} \cap E_{2}$. Then $\left\{\omega_{f^{\prime}(x)}\right\}$ and $\left\{\omega_{g^{\prime}(x)}\right\}$ are disjoint arrays which still witness $A \in A R E$ and $B \in A R E$ respectively. Then the disjoint array $\omega_{f^{\prime}(0)}, \omega_{g^{\prime}(0)}, \omega_{f^{\prime}(1)}, \omega_{g^{\prime}(1)} \cdots$ witnesses $A \cup B \in A R E$.

Lemma 3.6. Let $A$ be any nonrecursive set, and put $B=A$ join $N$. Then $B \notin A R$.

Proof. Suppose $B \in A R$ and let $F$ be a partial extension of $p_{B}^{-1}$. Then the following algorithm clearly decides whether $x \in B$ for any $x$ : if $x$ is odd, then $x \in B$; if $x$ is even calculate $F(x+1)$ and $F(x-1): x \in B$ iff $F(x+1)=F(x-1)+2$. Hence $B$ is recursive, but this is a contradiction since $A$ was not recursive.

Note if $A$ is any nonrecursive set in $A R E$, then $A$ join $N \in A R E-A R$. This simplifies and extends Theorem 3.2 of [7].

4. Almost finiteness properties. Theorem 3.2 clearly suggests that we study the relationships between $A R$ and the classes of immune sets listed at the end of $\S 1$. From Theorems 3.3 and 3.4 we have immediately:

THEOREM 4.1. (1) An infinite set $A$ is in SHHI iff it has no infinite ARE subset.

(2) If $A \in S H H I$ or $A \in S H I$ then $A \notin A R E$.

Since every hyperhyperimmune set with recursively enumerable complement is in $S H I$, such sets are never in $A R E$. P. R. Young has proved [9, Lemma 2.8] that FSHI sets with recursively enumerable complements are never in ARE. Yates' construction of a hyperhyperimmune retraceable set [8] shows that these implications may fail if the complement is not recursively enumerable since such a set is in $H H I, F S H I$, and $A R E$. We now give an alternate construction of such a set.

THEOREM 4.2. There exists a hyperhyperimmune set in ARE.

Proof. Let $f_{0}, f_{1}, \ldots$ be any nonrepetitive listing of all recursive functions which index finite disjoint arrays. Let $f$ be a recursive function such that

$$
\omega_{f(n)}=\left\{\operatorname{Pr}(n+1), \operatorname{Pr}(n+1)^{2}, \operatorname{Pr}(n+1)^{3}, \ldots\right\} .
$$

We define $A$ by stages and put:

$$
A^{(n)}=\{x \mid x \text { is listed in } A \text { by the end of stage } n\} .
$$

At the same time we define another set $B ; B^{(n)}$ is defined similarly and will contain elements which are ineligible to be placed in $A$ at any later stage, so that $B=\bigcup_{n=0}^{\infty} B^{(n)}$ will be a subset of $\bar{A}$.

Stage 0. Put $A^{(0)}=\{2\}$. Let $r_{0}=\mu n\left[2 \notin \omega_{f_{0}(n)}\right]$. Such a number $r_{0}$ must exist since $\left\{\omega_{f_{0}(n)}\right\}$ is a finite disjoint array. Put $B^{(0)}=\omega_{f_{0}\left(r_{0}\right)}$.

Stage $n+1$. Let $x=\mu y\left[y \in \omega_{f(n+1)} \wedge y \notin B^{(n)}\right]$. Put $A^{(n+1)}=A^{(n)} \cup\{x\}$. Let $r_{n+1}$ $=\mu k\left[\omega_{f_{n+1}(k)} \cap A^{(n+1)}=\varnothing\right]$. Since $A^{(n+1)}$ is a finite set, such a $k$ must exist. Now put $B^{(n+1)}=B^{(n)} \cup \omega_{f_{n+1}\left(r_{n+1}\right)}$. Finally, $A=\bigcup_{n=0}^{\infty} A^{(n)}$.

We note the following facts about the construction. At the end of stage $n, A^{(n)}$ and $B^{(n)}$ are both well defined finite sets so that our prescription for stage $n+1$ can 
actually be followed. It is clear from the construction that $\omega_{f(n)} \cap A$ is a singleton for each $n$. Also $A \subset \bigcup_{n \in N} \omega_{f(n)}$, and hence the disjoint array $\left\{\omega_{f(n)}\right\}$ is witness to the fact that $A \in A R E$. On the other hand, if $\left\{\omega_{g(x)}\right\}$ is any finite disjoint array, then $g=f_{i}$ for some $i$. Then by our construction we have $\omega_{f_{i}\left(r_{i}\right)} \cap A=\varnothing$, so that $\left\{\omega_{g(x)}\right\}$ cannot witness the nonhyperhyperimmunity of $A$. Hence $A$ is hyperhyperimmune.

COROLLARY 4.3. There exists a hyperhyperimmune retraceable set.

Proof. By Theorem 3.4 and the fact that every infinite subset of a hyperhyperimmune set is also hyperhyperimmune.

Definition 4.1. A set $A$ is called finitely almost recursively enumerable $(A \in F A R E)$ if $A$ is finite or if there is a recursive function $f$ such that $\left\{\omega_{f(x)}\right\}$ is a finite disjoint array satisfying

(i) $\bigwedge_{x}\left[\omega_{f(x)} \cap A\right.$ is a singleton], and

(ii) $A \subset \bigcup_{x \in N} \omega_{f(x)}$.

A set $A$ is called strongly finitely almost recursively enumerable $(A \in S F A R E)$ if $A$ is finite or if there exists a recursive function $f$ such that $\{D(f(x))\}$ is a discrete array satisfying

(i) $\bigwedge_{x}[D(f(x)) \cap A$ is a singleton], and

(ii) $A \subset \bigcup_{x \in N} D(f(x))$.

Clearly $S F A R E \subset F A R E \subset A R E ; \quad A \in F S H I \Rightarrow A \notin F A R E$, and $A \in H I \Rightarrow$ $A \notin S F A R E$. These relationships are all summarized in Figure 1.
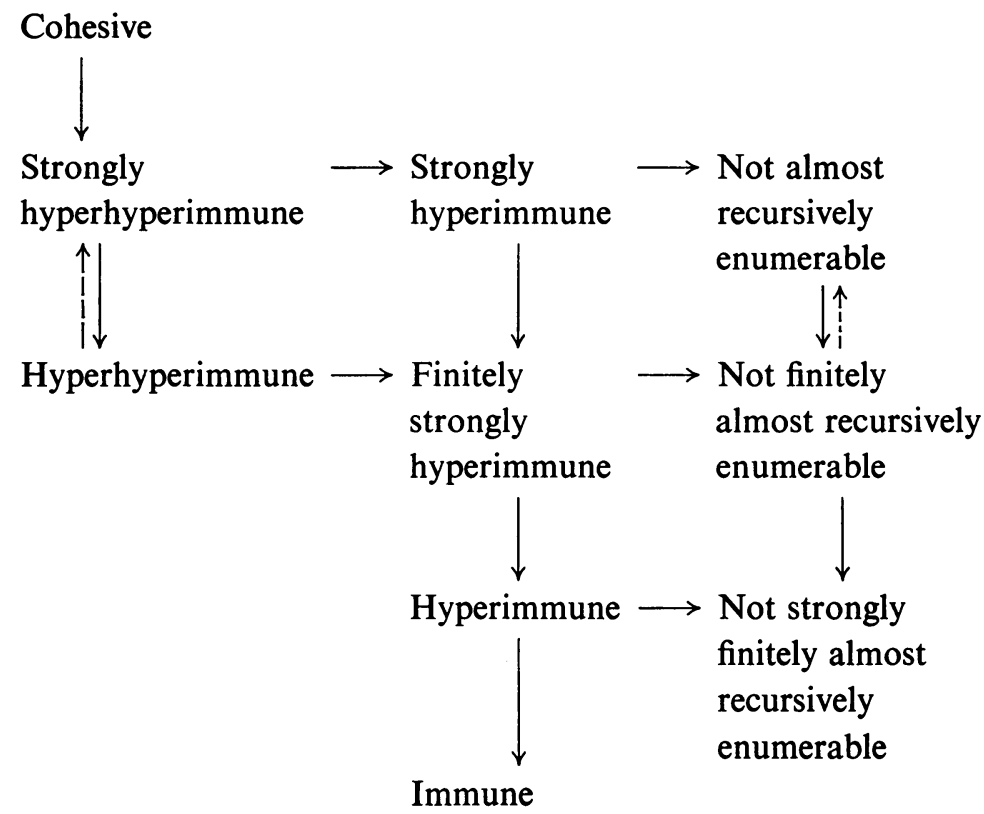

$\rightarrow$ indicates that the implication always holds.

$\rightarrow$ indicates that the implication holds if the complement is recursively enumerable, but fails in general. 
A set is immune iff it is infinite but has no infinite recursively enumerable subset. Using the classes $A R E, F A R E$, and SFARE, we can make the following analogous statements:

THEOREM 4.4. A set is hyperimmune iff it is infinite but has no infinite SFARE subset; a set is hyperhyperimmune iff it is infinite but has no infinite FARE subset; a set is strongly hyperhyperimmune iff it is infinite but has no infinite ARE subset.

Proof. If $A$ has a SFARE infinite subset, then there is a discrete array which meets $A$, so $A$ is not hyperimmune. Conversely, suppose $A$ is not hyperimmune. Then some discrete array $\{D(f(x))\}$ meets $A$. Put $E_{x}=\dot{D}(f(x)) \cap A$. Then each set $E_{x}$ is nonvoid. Picking exactly one element from each set $E_{x}$ produces an infinite $S F A R E$ subset of $A$. The second statement is proved similarly and the third is Theorem 4.1.

THEOREM 4.5. Let $A$ be an infinite set. Then $A \in F A R E$ if and only if $\bar{A}$ is the range of an injective total function a such that $a^{-1}$ has a finite to one partial recursive extension.

Proof. The proof is a straightforward variation on the proof of Theorem 3.2, so we omit it.

THEOREM 4.6. Let $A$ be an infinite set in ARE with $\bar{A}$ recursively enumerable. Then $A \in F A R E$.

Proof. This can be proved by a minor modification of the proof of Lemma 2.8 in [9] (of which our theorem is simply a slightly stronger version). We omit the details.

THEOREM 4.7. Let $A$ be an infinite almost recursive set. Then if either $A$ is recursively enumerable or $A$ is not hyperimmune, we have $A \in F A R E$.

Proof. If $\bar{A}$ is recursively enumerable, by Theorem 4.6, we have $A \in F A R E$ so let us assume that $A$ is not hyperimmune. Let $p_{A}$ be the principal function of $A$, and $F$ a partial recursive extension of $p_{A}^{-1}$. Since $A$ is not hyperimmune, there is a recursive function $\varphi$ such that $p_{A}(x) \leqq \varphi(x)$ for every $x$. Define a sequence of sets $E_{i}$ by

$$
x \in E_{i} \Leftrightarrow x \in \delta(F) \wedge F(x)=i \wedge x \leqq \varphi(i) .
$$

It is easily seen that the sets $E_{i}$ are finite and pairwise disjoint. The predicate $x \in E_{i}$ is recursively enumerable so there is a recursive function $f$ such that $E_{i}=\omega_{f(i)}$ for every $i$. Since $E_{i} \cap A=\left\{p_{A}(i)\right\}$ for each $i$, the finite disjoint array $\left\{\omega_{f(i)}\right\}$ witnesses the fact that $A \in F A R E$.

COROLlary 4.8. If $A$ is an infinite set which is in AR but not in FARE then $A$ is hyperimmune but not cohypersimple. (Such sets certainly exist: for example, a hyperhyperimmune retraceable set is in AR but cannot be in FARE.) 
THEOREM 4.9. Let $A \in S F A R E$ be an infinite set, with $\bar{A}$ recursively enumerable. Then $A$ is recursively enumerable (and hence recursive).

Proof. Let $f$ be a recursive function such that $\{D(f(x))\}$ is a discrete array witnessing $A \in S F A R E$. Let $r$ be a recursive function such that $r(x)=$ cardinality of $D(f(x))$ for every $x$. Let $g(x, t)$ be a partial recursive function of two variables such that

$$
D(f(x))=\{g(x, 0), g(x, 1), \ldots g(x, n)\}
$$

where $n=r(x)-1$. Then we have

$$
\begin{aligned}
x \in A \Leftrightarrow & \bigvee_{z}[x \in D(f(z)) \wedge r(z)=1] \\
& \vee \bigvee_{z}\left[x \in D(f(z)) \wedge \bigwedge_{i=0}^{r(z) \div 1}[g(z, i) \neq x \Rightarrow g(z, i) \in \bar{A}]\right] .
\end{aligned}
$$

Note that this predicate is recursively enumerable, so $A$ is a recursively enumerable set.

It would seem to follow from this theorem that $S F A R E$ is a rather restricted class of sets. However, by Theorem 2.2 every Turing degree contains a set $A$ such that both $A$ and $\bar{A}$ are in $S F A R E$.

5. Recursive equivalence types. We denote recursive equivalence (in the sense of Dekker \& Myhill [4]) by $\simeq$.

THEOREM 5.1. Let $A$ be an almost recursively enumerable set, and $A \simeq B$. Then $B$ is almost recursively enumerable.

Proof. If $A$ is finite the result is trivial. If $A$ is infinite let $a$ be an a.r. function with range $A$, and let $A \simeq B$ via the partial recursive function $\varphi$. Then $b(n)=\varphi(a(n))$ defines an almost recursive function with range $B$.

THEOREM 5.2. Let $A \in F A R E$ and $A \simeq B$. Then $B \in F A R E$.

Proof. Similar to that of 5.1 noting that if $a^{-1}$ has a finite-one partial recursive extension then so does $b=\varphi \circ a$.

Thus any R.E.T. which contains an $A R E$ (FARE) set, consists entirely of $A R E$ $(F A R E)$ sets. Dekker [2, Proposition 5a] proves that every regressive function is recursively equivalent to a strictly increasing regressive function, and hence that every regressive isol contains a retraceable set. In contrast to this we have:

THEOREM 5.3. There exists an almost recursive function which is not recursively equivalent to any strictly increasing (almost recursive) function.

Proof. We require the construction of a function $a(n)$ which is almost recursive and has the following property:

$\left(^{*}\right)$ For any partial recursive function $\varphi$ which is injective on its domain, either 
(i) $\bigvee_{n}[a(n) \notin \delta(\varphi)]$ or

(ii) $\bigvee_{n} \bigvee_{m}[n<m \wedge \varphi(a(m)) \leqq \varphi(a(n))]$

We first outline the basic strategy of the construction, which proceeds by stages. At the $n$th stage we define $a(n)$ although we reserve the right to change its value at later stages. We always pick $a(n)$ to be an element of the $n$th set of the disjoint array $\left\{\omega_{f(n)}\right\}$ where

$$
\omega_{f(n)}=\left\{\operatorname{Pr}(n+1), \operatorname{Pr}(n+1)^{2}, \operatorname{Pr}(n+1)^{3}, \ldots\right\} .
$$

This ensures that $a(n)$ will be an a.r. function. We also want $a(n)$ to have property $\left.{ }^{*}\right)$. To produce this, let $\varphi_{0}, \varphi_{1}, \varphi_{2}, \ldots$ be a list without repetitions of all partial recursive functions which are injective on their domains. We denote by $a^{n}(0)$, $a^{n}(1), \ldots, a^{n}(n)$ the values of $a$ (for the arguments $\left.0,1, \ldots, n\right)$ which are determined at the end of the $n$th stage.

Definition. We say that a number $k$ destroys $\varphi_{r}$ at the $n$th stage if $k \leqq n$ and $\varphi_{r}\left(a^{n}(k)\right)$ is undefined (i.e., $\left.a^{n}(k) \notin \delta\left(\varphi_{r}\right)\right)$. We say that a number pair $(k, m)$ destroys $\varphi_{r}$ at the $n$th stage if $k<m \leqq n$ and $\varphi_{r}\left(a^{n}(m)\right) \leqq \varphi_{r}\left(a^{n}(k)\right)$. Note that this notation means that both the numbers $a^{n}(m)$ and $a^{n}(k)$ belong to $\delta\left(\varphi_{r}\right)$ and that the inequality holds.

Our strategy is to ensure that $\varphi_{n}$ is destroyed by our actions at stage $n+1$, while taking care that $\varphi_{0}, \varphi_{1}, \ldots, \varphi_{n-1}$, which have been destroyed at earlier stages, should remain destroyed after our operations at stage $n+1$. Our construction is set up so that each function value $a(x)$ is changed at most twice, i.e. for each fixed $x$, the function $\alpha(n)=a^{n}(x)$ changes its value at most twice. So $a(x)$ is well defined as the final value of $a^{n}(x)$ or as $\lim _{n \rightarrow \infty} a^{n}(x)$.

We use systems of markers as an accounting procedure to keep track of the number or number pair which has been used to destroy each $\varphi_{r}$. If at any stage we have explicitly used the number $k$ to destroy $\varphi_{r}$, then at that stage $k$ will bear the marker $\boldsymbol{r}_{u}$. If at any stage we have explicitly used the number pair $(k, m)$ to destroy $\varphi_{r}$, with $k<m$, then $k$ will bear the marker $\boldsymbol{r}_{b}$ and $m$ the marker $\boldsymbol{r}_{a}$ at that stage. At a given stage a number may bear more than one marker; however at the beginning of stage $n+1$ it will be evident that the following situation holds: For each $r=0,1, \ldots, n-1$, either the single marker $\boldsymbol{r}_{u}$ or the marker pair $\boldsymbol{r}_{b}, \boldsymbol{r}_{a}$ but not both, is attached to numbers in the list $0,1, \ldots, n$. If the marker pair $\boldsymbol{r}_{b}, \boldsymbol{r}_{a}$ is attached, then $\boldsymbol{r}_{b}$ is always attached to a number strictly less than the number to which $\boldsymbol{r}_{a}$ is attached. If $y<x \leqq n$ are such that $\boldsymbol{r}_{b}$ is attached to $y$ and $\boldsymbol{r}_{a}$ to $x$ then the only other markers that $x$ and $y$ can bear are of type $s_{u}$ or else one other pair $s_{b}$ and $s_{a}$ with $s_{b}$ attached to $y$ and $s_{a}$ to $x$. No more than two such marker pairs can be attached at any given stage to any fixed pair of numbers. We now give explicit instructions for the construction.

Stage 0. Put $a^{0}(0)=2$ and proceed to Stage 1 .

Stage $n+1$. Our instructions involve a number of cases. They are to be followed in order, and the conditions for each case to occur implicitly include the negations 
of the conditions for the previous cases, since such an instruction is only reached if the conditions for the previous cases have not been fulfilled. At the beginning of the stage we have $a^{n}(0), a^{n}(1), \ldots, a^{n}(n)$ given, and $\varphi_{0}, \ldots, \varphi_{n-1}$ destroyed. Our objective is to destroy $\varphi_{n}$ while keeping $\varphi_{0}, \varphi_{1}, \ldots, \varphi_{n-1}$ destroyed.

Case A. $\bigvee_{k}\left[0 \leqq k \leqq n \wedge \varphi_{n}\left(a^{n}(k)\right)\right.$ is undefined $]$.

Then put $a^{n+1}(n+1)=\operatorname{Pr}(n+2) ; a^{n+1}(j)=a^{n}(j)$ for all $0 \leqq j \leqq n$. Place $n_{u}$ beside $\mu k\left(k \leqq n \wedge \varphi_{n}\left(a^{n}(k)\right)\right.$ is undefined). Proceed to stage $n+2$.

Case $\mathrm{B}_{1} . \bigvee_{y}\left[y \in \omega_{f(n+1)} \wedge \varphi_{n}(y)\right.$ is undefined].

Then put $a^{n+1}(n+1)=$ the smallest such $y ; a^{n+1}(j)=a^{n}(j)$ for $0 \leqq j \leqq n$. Place $n_{u}$ be side $n+1$ and proceed to stage $n+2$.

Case $\mathrm{B}_{2}$.

$\bigvee_{y}\left\{y \in \omega_{f(n+1)} \wedge \bigvee_{r \leqq n}[r\right.$ bears no marker at the end of stage $n$

$$
\left.\left.\wedge \varphi_{n}(y)<\varphi_{n}\left(a^{n}(r)\right)\right]\right\}
$$

Then put $a^{n+1}(n+1)=$ the smallest such $y ; a^{n+1}(j)=a^{n}(j)$ for $0 \leqq j \leqq n$. Let $r^{\prime}=$ $\mu r\left[r\right.$ bears no marker at the end of stage $\left.n \wedge \varphi_{n}\left(a^{n+1}(n+1)\right)<\varphi_{n}\left(a^{n}(r)\right)\right]$. Place $n_{b}$ beside $r^{\prime}$ and $n_{a}$ beside $n+1$. Proceed to stage $n+2$.

Case C. Set $a^{n+1}(n+1)=\operatorname{Pr}(n+2)$.

Subcase $\mathrm{C}_{1} . \bigvee_{r \leqq n}[r$ bears no marker at the end of stage $n]$. Fix the smallest such $r$. Then one of two things must happen.

Case $\mathrm{C}_{1,1} \cdot \bigvee_{y}\left[y \in \omega_{f(r)} \wedge \varphi_{n}(y)\right.$ is undefined]. Then put $a^{n+1}(r)=$ the smallest such $y$; and $a^{n+1}(j)=a^{n}(j)$ for $j \neq r, 0 \leqq j \leqq n$. Place $n_{u}$ beside $r$ and proceed to stage $n+2$.

Case $\mathrm{C}_{1,2} . \bigvee_{y}\left[y \in \omega_{f(r)} \wedge \varphi_{n}(y)>\varphi_{n}\left(a^{n+1}(n+1)\right)\right]$. Then put $a^{n+1}(r)=$ the smallest such $y$; and $a^{n+1}(j)=a^{n}(j)$ for $j \neq r, 0 \leqq j \leqq n$. Place $n_{b}$ beside $r$ and $n_{a}$ beside $n+1$. Proceed to stage $n+2$.

Subcase $\mathrm{C}_{2}$. This case occurs within Case $\mathrm{C}$ when every $r, 0 \leqq r \leqq n$, has at least one marker beside it at the end of stage $n$. Then by Lemma 1 (to follow) there is an $r$ such that $0 \leqq r \leqq n-1$, which satisfies the following conditions: $r_{b}$ is beside $y$, and $\boldsymbol{r}_{a}$ beside $x$ for some $y<x \leqq n$, and $y$ bears no other marker. Fix the smallest such $r$. Note that the corresponding arguments $x$ and $y$ have already been used to destroy $\varphi_{r}$ by having $\varphi_{r}\left(a^{n}(x)\right)<\varphi_{r}\left(a^{n}(y)\right)$. We use the same two arguments to destroy $\varphi_{n}$ while keeping $\varphi_{r}$ destroyed, by changing the value of $a^{n}(y)$. Four cases are possible.

Case $\mathrm{C}_{2,1} \cdot \bigvee_{z}\left[z \in \omega_{f(y)} \wedge \varphi_{r}(z)\right.$ is undefined $\wedge \varphi_{n}(z)$ is undefined]. Then put $a^{n+1}(y)=$ the smallest such $z$; and $a^{n+1}(j)=a^{n}(j)$ for $j \neq y, 0 \leqq j \leqq n$. Erase $\boldsymbol{r}_{a}$ and $\boldsymbol{r}_{b}$. Place $\boldsymbol{r}_{\boldsymbol{u}}$ and $\boldsymbol{n}_{\boldsymbol{u}}$ beside $y$, and proceed to stage $n+2$.

Case $\mathrm{C}_{2,2} \cdot \bigvee_{z}\left[z \in \omega_{f(y)} \wedge \varphi_{r}(z)\right.$ is undefined $\left.\wedge \varphi_{n}\left(a^{n}(x)\right)<\varphi_{n}(z)\right]$. Then put $a^{n+1}(y)=$ the smallest such $z ; a^{n+1}(j)=a^{n}(j)$ for $j \neq y, 0 \leqq j \leqq n$. Erase $\boldsymbol{r}_{b}$ and $\boldsymbol{r}_{a}$. Place $\boldsymbol{r}_{u}$ beside $y$, and place $n_{b}$ beside $y$ and $n_{a}$ beside $x$. Then proceed to stage $n+2$. 
Case $\mathrm{C}_{2,3} . \bigvee_{z}\left[z \in \omega_{f(y)} \wedge \varphi_{r}\left(a^{n}(x)\right)<\varphi_{r}(z) \wedge \varphi_{n}(z)\right.$ is undefined]. Then put $a^{n+1}(y)$ $=$ the smallest such $z ; a^{n+1}(j)=a^{n}(j)$ for $j \neq y, 0 \leqq j \leqq n$. Place $n_{u}$ beside $y$, and proceed to stage $n+2$.

Case $\mathrm{C}_{2,4} . \bigvee_{z}\left[z \in \omega_{f(y)} \wedge \varphi_{r}\left(a^{n}(x)\right)<\varphi_{r}(z) \wedge \varphi_{n}\left(a^{n}(x)\right)<\varphi_{n}(z)\right]$. Then put $a^{n+1}(y)$ $=$ the smallest such $z ; a^{n+1}(j)=a^{n}(j)$ for $j \neq y, 0 \leqq j \leqq n$. Place $n_{b}$ beside $y$ and $\boldsymbol{n}_{a}$ beside $x$. Then proceed to stage $n+2$.

This completes the description of the construction.

LEMMA 1. If at the beginning of stage $n+1$, every number in the list $0,1, \ldots, n$ bears at least one marker, then there are numbers $x$ and $y$ with $0 \leqq y<x \leqq n$ such that $y$ bears a marker $\boldsymbol{r}_{b}, x$ a marker $\boldsymbol{r}_{a}$ for some $r \leqq n-1$, and $y$ bears no other marker.

Proof. By examination of the procedures followed at stage $n+1$ we note that a marker pair $\boldsymbol{n}_{b}, \boldsymbol{n}_{a}$ can only be introduced in the following ways:

(a) Beside blank numbers, via Case $\mathrm{B}_{2}$ or Case $\mathrm{C}_{1,2}$, in which case, $\boldsymbol{n}_{a}$ is placed beside $n+1$.

(b) Beside a pair $y<x$ which already bears a marker pair $\boldsymbol{r}_{b}, \boldsymbol{r}_{a}$ and $y$ bears no other marker, via Case $\mathrm{C}_{2,4}$.

(c) Beside a pair $y<x$ such that $y$ bears only a marker of the type $\boldsymbol{r}_{u}$ for some $r \leqq n-1$, via Case $\mathrm{C}_{2,2}$.

Now consider the numbers $0,1, \ldots, n$ and the markers they bear at the beginning of stage $n+1$. For each $r, 0 \leqq r \leqq n-1$, either the single marker $\boldsymbol{r}_{u}$, or the marker pair $\boldsymbol{r}_{b}, \boldsymbol{r}_{a}$ is attached in the list $0,1, \ldots, n$. We prove the lemma by induction on $n$. It is clearly true at the beginning of stage 2 . As inductive hypothesis assume that it is true for such systems of at most $n-1$ markers or marker pairs attached in the list $0,1, \ldots, n-1$ (as at the beginning of stage $n$ ). We divide the situation at the beginning of stage $n+1$ into two cases.

Case 1. $n$ bears the marker $(n-1)_{u}$. Then if we delete this marker, the remaining $n-1$ markers or marker pairs cover the numbers $0,1, \ldots, n-1$. By the inductive hypothesis there is a number $y<n-1$ which bears a single marker of the type $\boldsymbol{r}_{b}$ for some $r \leqq n-2$.

Case 2. $n$ bears the marker $(n-1)_{a}$. Then let $(n-1)_{b}$ be attached to the number $y \leqq n-1$. If $y$ bears no other marker we are finished. Otherwise, delete the marker pair $(n-1)_{b}$ and $(n-1)_{a}$. The remaining $n-1$ markers or marker pairs cover the numbers $0,1, \ldots, n-1$ so we can apply the inductive hypothesis.

LEMMA 2. For each $x$, as $n$ increases, $a^{n}(x)$ changes its value at most twice.

Proof. We note that $a^{n+1}(x) \neq a^{n}(x)$ only via the procedures of Case $\mathrm{C}$, when $x$ (at the beginning of stage $n+1$ ) either bears no marker at all or else a single marker of type $\boldsymbol{r}_{b}$. A straightforward examination of the way markers are assigned in Case $\mathrm{C}$ reveals the lemma, since after two changes in the value of $a(x), x$ cannot bear either of these configurations of markers.

We now complete the proof of the theorem. By Lemma 2, $a(x)=$ the final value of $a^{n}(x)$ is well defined for every $x$. Our construction was set up so that, for every $x$, 
$a(x) \in \omega_{f(x)}$, and $\left\{\omega_{f(x)}\right\}$ is a disjoint array. Then by Theorem 4.2, $a$ is an almost recursive function. Let $b$ be any function such that $a$ is recursively equivalent to $b$. Then for some $i \in N$, we have $b(n)=\varphi_{i}(a(n))$ for every $n$. But our construction ensures that whenever $\delta\left(\varphi_{i}\right) \supset \rho(a)$ (i.e., $\varphi_{i} \circ a$ is total) then $\varphi_{i} \circ a$ is not strictly increasing. Hence $a$ is not recursively equivalent to any strictly increasing function, and our theorem is proved.

Theorem 6.3 does not in itself enable us to conclude that there is an $A R E$ set which is not recursively equivalent to any $A R$ set. The missing step is the theorem that if $a$ and $b$ are a.r. functions, with $\rho(a) \simeq \rho(b)$ then $a \simeq b$. This is true for regressive functions [2, Proposition 3], but the following construction, suggested by the referee, shows that it fails to hold for almost recursive functions.

THEOREM 6.4. (Suggested by the referee.) There exist a.r. functions $a_{1}$ and $a_{2}$ such that $\rho\left(a_{1}\right)=\rho\left(a_{2}\right)$ but $a_{1} \not a_{2}$. Moreover $A=\rho\left(a_{1}\right)$ is SFARE.

Proof. Let $\left\{\varphi_{n}\right\}$ be the list of one-to-one partial recursive functions as in 6.3. Let $\{D(g(x))\}$ be a discrete array such that every set $D(g(x))$ contains exactly three elements. Define $a_{1}$ and $a_{2}$ by stages so that

(1) $A=\rho\left(a_{1}\right)=\rho\left(a_{2}\right) \subset \bigcup_{x=0}^{\infty} D(g(x))$,

(2) $\bigwedge_{x} A \cap D(g(x))$ is a singleton,

(3) $\wedge_{x}\left[a_{1}(2 x) \in D(g(2 x)) \wedge a_{2}(2 x+1) \in D(g(2 x))\right]$,

(4) $\bigwedge_{x}\left[a_{1}(2 x+1) \in D(g(2 x+1)) \wedge a_{2}(2 x) \in D(g(2 x+1))\right]$,

(5) $a_{1} \not a_{2}$, i.e. $\bigwedge_{n}\left[A \subset \delta\left(\varphi_{n}\right) \Rightarrow \bigvee_{x}\left[\varphi_{n}\left(a_{1}(x)\right) \neq a_{2}(x)\right]\right]$.

Then $a_{1}$ and $a_{2}$ clearly satisfy the conditions of the theorem. The procedure at the $n$th stage is as follows. Order $D(g(2 n)) \times D(g(2 n+1))$ lexicographically. Let $\langle m, k\rangle$ be the least pair in $D(g(2 x)) \times D(g(2 x+1))$ such that $\varphi_{n} \cap\{\langle m, k\rangle,\langle k, m\rangle\}=\varnothing$. Since $\varphi_{n}$ is one-to-one and there are nine pairs to choose from, such a pair $\langle m, k\rangle$ clearly exists. Put $a_{1}(2 n)=m=a_{2}(2 n+1)$ and $a_{1}(2 n+1)=k=a_{2}(2 n)$. Then conditions (1) to (5) are clearly satisfied.

Finally, we note that Proposition 5(b) of [2] does fail in the almost recursive case.

THEOREM 6.5. There is an almost recursively enumerable set which is not recursively equivalent to any almost recursive set.

Proof. Let $A$ be any nonimmune set which is almost recursively enumerable, but not finitely almost recursively enumerable. (For example, let $B$ be a hyperhyperimmune retraceable set and let $A=B$ join $N$.) Then $A$ satisfies the conditions of the theorem. For suppose $A \simeq C$ and $C$ is almost recursive. By Theorem 5.2, $C \notin F A R E$, but $C$ is not hyperimmune and hence, by Theorem 4.7, $C \in F A R E$. Thus $A \simeq C$ implies $C$ is not almost recursive.

We may note that any a.r. function with range $A$ gives another example of a function satisfying the conditions of Theorem 6.3. Nevertheless the explicit construction technique described in that theorem appears to be of independent interest. 


\section{REFERENCES}

1. K. I. Appel and T. G. McLaughlin, On properties of regressive sets, Trans. Amer. Math. Soc. 155 (1965), 83-93. MR 37 \#6176.

2. J. C. E. Dekker, Infinite series of isols, Proc. Sympos. Pure Math., vol. 5, Amer. Math. Soc., Providence, R. I., 1962, pp. 77-96. MR 26 \#16.

3. J. C. E. Dekker and J. Myhill, Retraceable sets, Canad. J. Math. 10 (1958), 357-373. MR 20 \#5733.

4. - Recursive equivalence types, Univ. California Publ. Math. 3(1960), 67-213. MR 22 \#7938.

5. T. G. McLaughlin, Strong reducibility on hypersimple sets, Notre Dame J. Formal Logic 6 (1965), 229-234. MR 33 \#1234.

6. H. Rogers, Jr., Theory of recursive functions and effective computability, McGraw-Hill, New York, 1967. MR 37 \#61.

7. V. D. Vuckovic, Almost recursive sets, Proc. Amer. Math. Soc. 23 (1969), 114-119. MR 41 \#5217.

8. C. E. M. Yates, Recursively enumerable sets and retracing functions, Z. Math. Logik Grundlagen Math. 8 (1962), 331-345. MR 26 \#3598.

9. P. R. Young, Linear orderings under one-one reducibility, J. Symbolic Logic 31 (1966), 70-85. MR 38 \#48.

Department of Mathematics and Astronomy, The University of Manitoba, Winnipeg, Manitoba, Canada 